Gut, 1971, 12, 119-122

\title{
Trends in mortality from ulcerative colitis in England and Wales
}

\section{J. GRIMLEY EVANS}

From the Department of Medical Statistics and Epidemiology, London School of Hygiene and Tropical Medicine

SUMMARY Mortality rates for ulcerative colitis in England and Wales over the years 1946 to 1967 showed trends which varied with age. Between the ages of 15 and 34 rates fell with only minor irregularities. At ages 35 to 64, rates were constant from 1946 to 1957 and then began to decline. At ages 65 and above, rates rose during most of the period but with a recent tendency to stabilize. These trends are discussed in relation to possible effects of the introduction of steroids and other therapies.

There have been important developments in the treatment of ulcerative colitis in the last two decades. Steroids became available in the early 1950 s and their efficacy in treating ulcerative colitis was established in the clinical trial by Truelove and Witts (1955). Sulphasalazine had been used in Scandinavia and the United States since about 1941, but the results of the first controlled clinical trials were published in 1962 (Baron, Connell, Lennard-Jones, and Jones, 1962; Truelove, Watkinson, and Draper, 1962). Indications for surgical treatment and the management of surgical patients were also being refined during the period, There is, however, no way of knowing how rapidly and effectively these various innovations came into general use.

Crude mortality rates of ulcerative colitis in England and Wales responded little to these therapeutic advances. This might suggest that the new treatments had little impact on fatality, but a possible complicating factor is that the disease may have become commoner (Acheson, 1965). Crude rates conceal some variations with age in the temporal trend of mortality that are of interest in this context.

\section{Material}

From 1940 until 1949 ulcerative colitis was coded according to the Fifth Revision of the International List of Causes of Death under '119(b) and 120(b), ulceration of the intestines (except duodenum)'. This category was distinguished from other forms of enteritis and diarrhoea without mention of ulceration. The Sixth Revision of the International Statistical Classification of Diseases, Injuries and

Received for publication 6 October 1970.
Causes of Death (ISCD) was brought into use in England and Wales in 1950. Under this revision 'ulcerative colitis' was given a specific category, number 572.2. Double coding of the 1949 deaths showed that the effect of the Sixth Revision was to reduce the number of deaths allotted to the category of ulcerative colitis by about $17 \%$, but the effect was greater in older age groups than in younger (Registrar General, 1953). The Seventh Revision of the ISCD, first used in England and Wales in 1958, had a negligible effect on the number of deaths ascribed to ulcerative colitis (Registrar General, 1959). Category 572.2 of the Sixth and Seventh Revisions included any form of enteritis specified as ulcerative in persons aged four weeks or more, but excluding specific conditions such as Crohn's disease and synonyms (coded to 572.0) or diverticulitis (572.1). Colitis not specified as chronic or ulcerative was assigned to category 571. Diagnoses of 'mucous colitis', 'colitis gravis', and 'diarrhoea' were assignable from 1950 to other categories (573, $316.0,785.6$ ) but these were very rarely nominated as the underlying cause of death.

\section{Results}

Figure 1 shows age-standardized mortality rates for ulcerative colitis (572.2) for males and females from 1940 until 1967. In both sexes rates fell on average during the war years and were fairly stable for about a decade after 1947. From 1958 there has been a downward trend. In females, the mean rate between 1963 and 1967 was $24 \%$ lower than that between 1953 and 1957. For males corresponding figures show a $14 \%$ decrease.

Mortality rates for ulcerative colitis are very small 


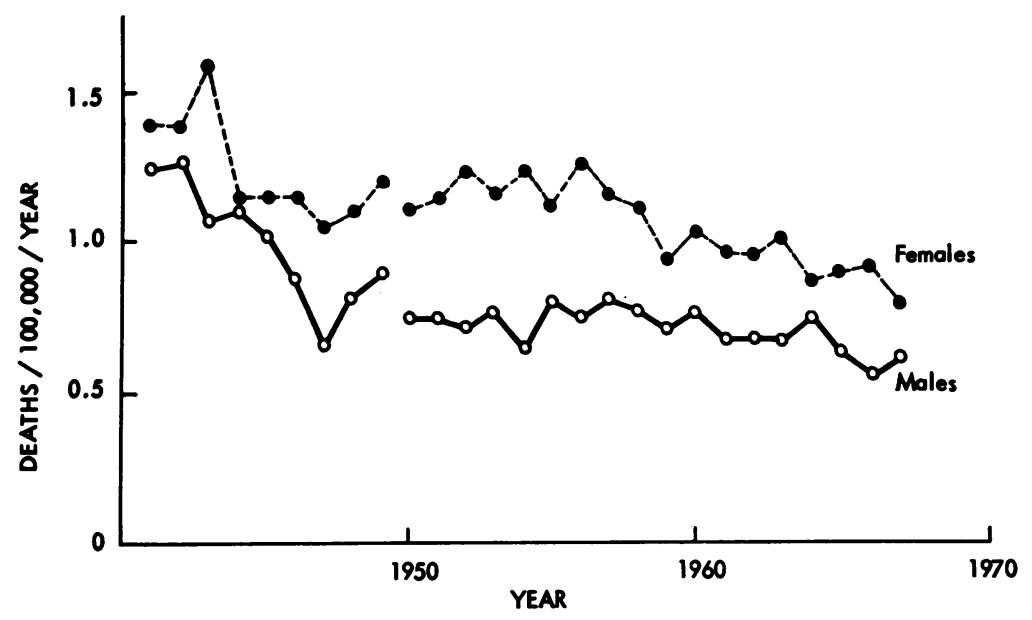

Fig. 1. Annual mortality rates for ulcerative colitis in England and Wales, standardized by five-year age groups to the 1951 Census population. The gaps in the curves indicate coding revision. at ages 1 to 14 years. For older age groups, sexspecific annual mortality rates for two-year periods from 1946 to 1967 are shown in the Table. (Rates for the war years 1940-1945, were unstable as can be judged from Fig. 1, and there is doubt about the effective population at risk during this period.) In several of the groups analysed in the Table-particularly in males-rather small numbers are involved, so that the interpretation of individual rates should be cautious. Overall, the female mortality rates are higher than male, as are incidence and prevalence rates (Evans and Acheson, 1965). There is a suggestion that below the age of 35 the male/female ratio has lately increased while among the elderly, aged 75 and over, a female preponderance did not emerge until the mid-1950s. Owing to the small numbers in these two age groups it is difficult to be sure of these trends. At other ages the sex differential has been generally maintained over the 20 years considered.
Temporal trends at different ages are of similar pattern in the two sexes. Among young adults, aged 15 to 34, rates fell with only minor irregularities throughout the 20-year period. The elderly, aged 65 and over, showed increasing rates through the period with some recent tendency to stabilize. The intermediate age groups aged 35 to 64 , having been fairly constant from 1946, began to decline from 1958 . These trends are smoothed and epitomized in Fig. 2 by combining the age and sex groups into standardized rates.

Death rates for Crohn's disease (572.0) increased throughout the period from 1950 when the condition was first coded. The increase occurred for both sexes and most age groups over 14 years. Owing to the comparative rarity of the disease, the addition of deaths from Crohn's disease to those from ulcerative colitis has no effect on the trends shown in Figure 2.

Death rates for nonspecific gastroenteritis and

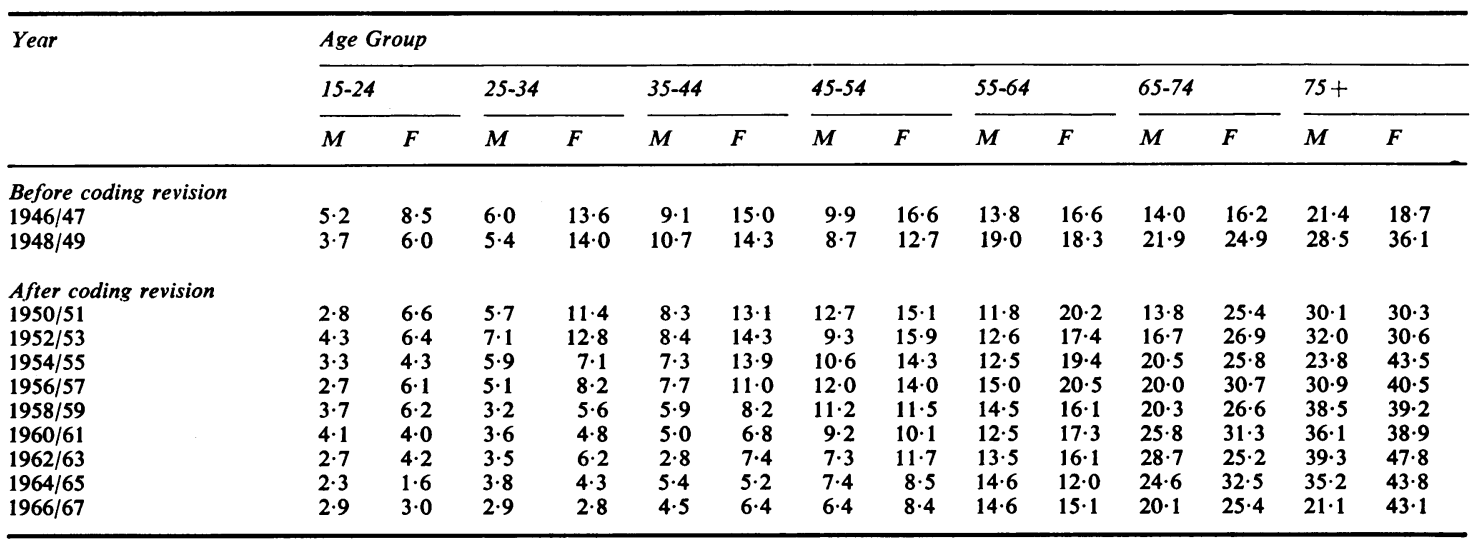

Table Annual mortality rates per million for two-year periods 1946-1967 by sex and age group 


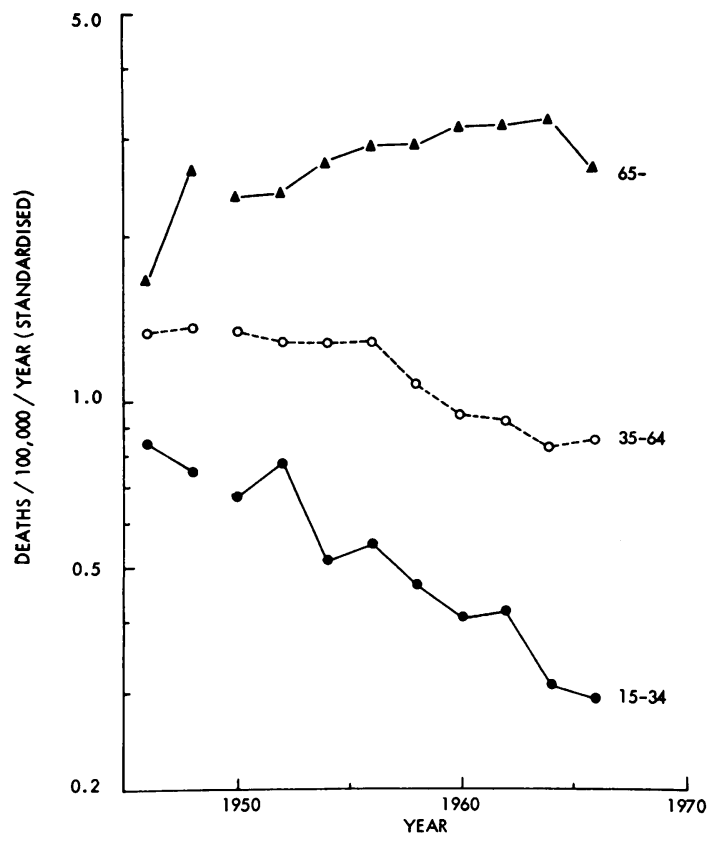

Fig. 2 Average annual mortality rates for ulcerative colitis in England and Wales for two-year periods 1946 to 1967 standardized for sex and age (by five-year groups) to the 1951 Census population. The gaps in the curves indicate coding revision.

colitis (category 571 of the Sixth and Seventh Revisions of the International Statistical Classification) were very low at ages 15 to 34 and showed no consistent trend from 1950 to 1967 . For the age group 35 to 64 , death rates fell with no obvious change of slope throughout the period. At ages 65 and above rates fell consistently from 1950 until about 1958 since when they have been constant. In this oldest age group the death rates for categories 571 and 572.2 combined was constant from 1950 to 1967 , but in the two younger age groups the combined death rates showed the same features as category 572.2 alone. For diverticulitis (572.1) death rates showed no consistent trends at ages under 65 but in older persons rose until 1961 and then stabilized. Other categories of diarrhoea or colitis $(573,316,785.6)$ contained too few deaths throughout the period for meaningful analysis.

\section{Discussion}

The inadequacies of conventional mortality statistics, based on the selection of a single 'underlying cause of death', and their sensitivity to changes in diag- nostic practice are well known. Taken in isolation, they cannot be expected to provide a reliable assessment of new therapy for a chronic disease such as ulcerative colitis. Although most people who develop some degree of the disease will be liable to symptoms throughout their lives, only a minority will ultimately die from it. The case-fatality ratio of ulcerative colitis (the mortality rate expressed as a proportion of the incidence rate) is between 0.2 and 0.1 . A further problem is the rarity of the disease. In order to provide adequate numbers for stable rates, data have to be pooled over time and across age and sex groups thereby blurring, and perhaps distorting, the effects of these variables.

In terms of fatality, the essential characteristic of improved therapy will be a prolongation of average true survival time between the onset of disease and death. It may not be easy to predict the dynamic relation between mean survival time and mortality rates (Stocks, 1935). It will depend partly on the variation of incidence with age, and also on the probability, relative to that of the general population, of a patient's death being attributed to some other cause. To take an extreme case, for a disease with equal incidence at all ages and no general effect on health, an increase in mean survival time would have a disproportionately small effect on mortality rates. The patients dying at a particular age would have had the disease for longer than their predecessors, but there would be no fewer of them. At the other extreme, a treatment could reduce mortality rates without incieasing survival time by 'transferring' deaths to other causes.

There are several reasons why national mortality data are an important complement to clinical trials and follow-up studies. First, they may provide the only indicator of whether effective treatment is reaching the people who need it. Second, clinical trials are carried out on selected patients in specialist centres and the effects of a more general use of the remedy cannot be safely predicted, as national mortality data have recently demonstrated for asthma (Inman and Adelstein, 1969). Third, survival times usually have to be measured from the time of diagnosis rather than from the onset of a disease. A tendency to earlier diagnosis following the interest aroused by a new treatment will by itself produce a lengthening of survival times measured in this way but, other things being equal, will not disturb agespecific mortality rates. Despite their defects, therefore, mortality data provide a necessary adjunct to clinical assessments of therapy.

For ulcerative colitis, the mortality figures for England and Wales suggest that some influence introduced in the second half of the 1950s initiated a decline in the previously steady mortality rates 
among people aged, approximately, 35 to 64 . The decline is not attributable to transfer of deaths to Crohn's disease, diverticulitis, or nonspecific colitis. The effect could well have been due to steroid therapy initially, but later developments in treatment may also be involved. The effect was not dramatic, but it occurred despite increasing diagnosis of the disease and probably in the face of increasing incidence (Evans and Acheson, 1965).

Among the young (ages 15 to 34 ), rates fell continuously from 1946 and any additional benefit conferred by the therapeutic advances of 1955 and after was obscured by pre-existing improvement attributable, perhaps, to control of infections and to growing knowledge of fluid and electrolyte physiology with safer surgery. Again, the trend is not explicable in terms of transfer of deaths to the categories of Crohn's disease or nonspecific colitis.

At ages 65 and above, rates rose throughout most of the period. This may reflect increasing recognition of the disease in this age group; from 1950 to 1958 there was a corresponding fall in the death rates from nonspecific colitis and enteritis (category 571). Since 1958 there has presumably been increased survival of patients from younger age groups as an additional factor.

It is important to note, from the Table, that the onset of declining rates in the age range 35 to 64 occurred at about the same time in its component groups. The trend shown in Fig. 2 might otherwise suggest that already from the 1940s patients with ulcerative colitis were surviving into successively older age groups, or that a cohort of persons with high susceptibility to the disease was born between 1880 and 1920.
Although the fall in death rates from ulcerative colitis in people aged under 65 is not attributable to the transfer of deaths to the categories of Crohn's disease, diverticulitis, or nonspecific colitis, there remains inevitably the disquieting possibility of transfer to other conditions occurring as complications of treatment. Without the special aid of medical record linkage or multiple cause coding of death certificates, this process could be extremely difficult to detect, since it might occur only after a new treatment has come into general use under conditions different from those of its original clinical trials.

I am grateful to Dr J. W. Donovan for helpful comments.

\section{References}

Acheson, E. D. (1965). The epidemiology of ulcerative colitis and regional enteritis. In Recent Advances in Gastroenterology, edited by J. Badenoch and B. N. Brooke, pp. 202-226. Churchill, London.

Baron, J. H., Connell, A. M., Lennard-Jones, J. E., and Jones, F. A. (1962). Sulphasalazine and salicylazosulphadimidine in ulcerative colitis. Lancet, 1, 1094-1096.

Evans, J. G., and Acheson, E. D. (1965). An epidemiological study of ulcerative colitis and regional enteritis in the Oxford area. Gut, 6, 311-324.

Inman, W. H. W., and Adelstein, A. M. (1969). Rise and fall of asthma mortality in England and Wales in relation to use of pressurised aerosols. Lancet, 2, 279-285.

Registrar General (1953). Statistical Review of England and Wales for the two Years 1948-1949. Text, Medical. HMSO, London.

Registrar General (1959). Statistical Review of England and Wales for the Year 1957. Pt. III: Commentary. HMSO, London.

Stocks, P. (1935). The lengthening of life by modern therapy in pernicious anaemia and diabetes. Brit. med. J., 1, 1013-1017.

Truelove, S. C., Watkinson, G., and Draper, G. (1962). Comparison of corticosteroid and sulphasalazine therapy in ulcerative colitis. Brit. med. J., 2, 1708-1711.

Truelove, S. C., and Witts, L. J. (1955). Cortisone in ulcerative colitis. Final report on a therapeutic trial. Brit. med. J., 2. 1041-1048. 